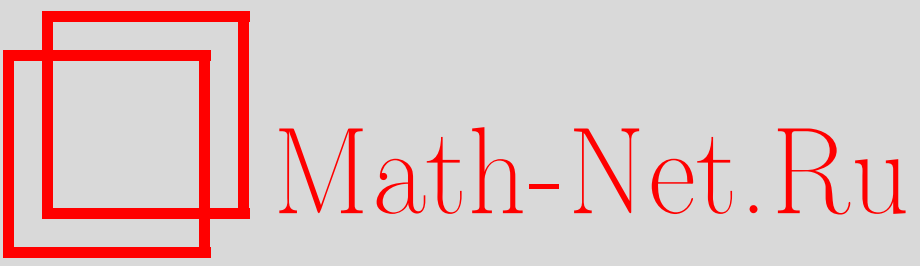

В. В. Тимошенко, К сходимости в смысле Чезаро числовых рядов, Итоги науки и техн. Сер. Соврем. мат. и ее прил. Темат. обз., 2020, том 179, 78-80

DOI: https://doi.org/10.36535/0233-6723-2020-179-78-80

Использование Общероссийского математического портала Math-Net.Ru подразумевает, что вы прочитали и согласны с пользовательским соглашением

http://www.mathnet.ru/rus/agreement

Параметры загрузки:

IP: 54.89 .56 .158

26 апреля 2023 г., 18:21:28 


\title{
К СХОДИМОСТИ В СМЫСЛЕ ЧЕЗАРО ЧИСЛОВЫХ РЯДОВ
}

\author{
(c) 2020 г. $\quad$ В. В. ТИМОШЕНКО
}

\begin{abstract}
АннотАция. Переход от заданного числового ряда к ряду усредненных сумм его членов называется процедурой Чезаро. В данной работе рассматривается ряд, для которого $n$-кратное применение процедуры Чезаро дает расходящиеся ряды, а $(n+1)$-кратное приводит к сходящемуся ряду.
\end{abstract}

Ключевые слова: числовой ряд, сходимость ряда, сходимость ряда в смысле Чезаро.

\section{ON THE CESÀRO CONVERGENCE OF NUMERICAL SERIES}

\section{(C) 2020 V. V. TIMOSCHENKO}

Abstract. The transition from a given series to the series of averaged sums of its terms is called the Cesàro procedure. In this paper, we construct a series for which $n$-multiple application of the Cesàro procedure gives divergent series whereas the $(n+1)$-multiple leads to a convergent series.

Keywords and phrases: series, convergence, Cesàro convergence.

AMS Subject Classification: 40A05, 40B05, 40F05, 40G05

Дан числовой ряд

$$
a_{1}+a_{2}+\ldots+a_{n}+\ldots,
$$

составленный из действительных или комплексных чисел. Будем называть переходом $C(1)$ переход от этого ряда к ряду, $n$-й член которого $(n=1,2,3, \ldots)$ равен среднему арифметическому $n$ первых членов исходного числового ряда:

$$
a_{n}^{1}=\frac{a_{1}+a_{2}+\ldots+a_{n}}{n} .
$$

Для ряда

$$
a_{1}^{1}+a_{2}^{1}+\ldots+a_{n}^{1}+\ldots
$$

аналогичную процедуру назовем переходом $C(2)$, она приводит нас к ряду

$$
a_{1}^{2}+a_{2}^{2}+\ldots+a_{n}^{2}+\ldots,
$$

составленному из средних арифметических первых $n$ членов ряда $(1)$, и т. д. Аналогично, переходом $C(k)$ назовем переход от ряда $(k-1)$

$$
a_{1}^{(k-1)}+a_{2}^{(k-1)}+\ldots+a_{n}^{(k-1)}+\ldots
$$

к ряду $(k), n$-й член которого $(n=1,2,3, \ldots)$ равен среднему арифметическому $n$ первых членов ряда $(k-1)$ :

$$
a_{n}^{(k)}=\frac{a_{1}^{(k-1)}+a_{2}^{(k-1)}+\ldots+a_{n}^{(k-1)}}{n} .
$$

По определению, ряд

$$
a_{1}+a_{2}+\ldots+a_{n}+\ldots
$$




\begin{tabular}{|c|c|c|c|c|c|c|c|c|c|c|c|c|c|c|c|c|c|c|}
\hline № & 1 & 2 & 3 & 4 & 5 & 6 & 7 & 8 & 9 & 10 & 11 & 12 & 13 & 14 & 15 & 16 & 17 & $\ldots$ \\
\hline 2 & 1 & 0 & 0 & $1 / 4$ & 0 & 0 & 0 & $1 / 8$ & 0 & 0 & 0 & 0 & 0 & 0 & 0 & $1 / 16$ & 0 & $\ldots$ \\
\hline 3 & 1 & -1 & 0 & 1 & -1 & 0 & 0 & 1 & -1 & 0 & 0 & 0 & 0 & 0 & 0 & 1 & -1 & $\ldots$ \\
\hline 4 & 1 & -3 & 2 & 4 & -9 & 5 & 0 & 8 & -17 & 9 & 0 & 0 & 0 & 0 & 0 & 16 & -33 & $\ldots$ \\
\hline 5 & 1 & -7 & 12 & 10 & -61 & 75 & -30 & 64 & -217 & 243 & -90 & 0 & 0 & 0 & 0 & 256 & -817 & $\ldots$ \\
\hline 6 & 1 & -15 & 50 & $\ldots$ & $\ldots$ & $\ldots$ & & & & & & & & & & & $\ldots$ & \\
\hline 7 & 1 & -31 & 180 & $\ldots$ & $\ldots$ & $\ldots$ & & & & & & & & & & & $\ldots$ & \\
\hline & & & & & & & & & & & & & & & & & \\
\hline$(k-1)$ & 1 & $a_{(k-1) 2}$ & $a_{(k-1) 3}$ & $a_{(k-1) 4}$ & $a_{(k-1) 5}$ & $\ldots$ & & & & & & & & & & & $\ldots$ & \\
\hline$k$ & 1 & $\ldots$ & $\ldots$ & $\ldots$ & $\ldots$ & $\ldots$ & & & & & & & & & & & $\ldots$ & \\
\hline
\end{tabular}

называется сходящимся в смысле Чезаро, если переход $C(1)$ дает ряд (1), сходящийся в обычном смысле.

Известно, что любой сходящийся ряд будет сходящимся в смысле Чезаро, причем к той же сумме. Обратное утверждение неверно. Ряд

$$
1+(-1)+0+1+(-1)+0+0+\ldots+0+1+(-1)+0+0+\ldots,
$$

где 1 и $(-1)$ стоят на 1-м и 2-м, 4-м и 5-м, 8-м и 9-м, 16-м и 17-м и т. д. местах, а все остальные члены ряда равны 0, сходится в смысле Чезаро, поскольку переход $C(1)$ дает ряд

$$
1+0+0+\frac{1}{4}+0+0+0+\frac{1}{8}+0+0+\ldots+0+\frac{1}{16}+0+\ldots,
$$

который сходится в обычном смысле (его сумма равна $3 / 2$ ). Но в обычном смысле он не является сходящимся хотя бы потому, что не выполняется необходимый признак сходимости числового ряда (его общий член не стремится к нулю при $n \rightarrow \infty)$ ).

Задача, поставленная доцентом кафедры математического анализа МГПИ им. В. И. Ленина Н. Д. Гиленко в 1972 году, заключалась в следующем. Требовалось построить такой числовой ряд, чтобы он сам расходился в смысле Чезаро, полученный из него переходом $C(1)$ ряд (1) тоже расходился в смысле Чезаро, такими же были ряды $(2),(3), \ldots$, полученные при помощи переходов $C(2), C(3), \ldots, C(N-1)$, а следующий ряд $(N)$, полученный при помощи перехода $C(N)$, был бы сходящимся в смысле Чезаро.

Поступаем следующим образом. Берем ряд (8), который не сходится в обычном смысле, но сходится в смысле Чезаро. Построим следующую таблицу. Первая строка таблицы - номера членов ряда: $1,2,3, \ldots$. Третья строка - записанные по порядку члены ряда (8), вторая строка - ряд, полученный из этого ряда переходом $C(1)$.

$\mathrm{B}$ четвертой строке записываем ряд, из которого переходом $C(1)$ получается ряд, записанный в третьей строке. Члены ряда

$$
a_{41}+a_{42}+\ldots+a_{4 n}+\ldots
$$

(символ $a_{4 n} 9$ означает $n$-й член ряда, записанного в 4-й строке) находятся по формулам

$$
a_{41}=1 ; \quad a_{4 n}=a_{3 n} \cdot n-\left(a_{41}+a_{42}+\ldots+a_{4(n-1)}\right), n>1 .
$$

Для 5-й строки, где записываем члены ряда, из которого переходом $C(2)$ получается ряд, записанный в 3-й строке (или, что то же самое, из которого переходом $C(1)$ получается ряд, записанный в 4-й строке), верны эти же формулы, только индекс 4 заменен на 5 , а 3 - на 4.

Вообще, для $k$-й строки, где будем записывать члены ряда, из которого переходом $C(k-3)$ получается ряд, записанный в 3 -й строке, или, что то же самое, из которого переходом $C(1)$ получается ряд, записанный в $(k-1)$-й строке, будут справедливы те же формулы, но где индекс 4 заменен на $k$, а индекс 3 - на $(k-1)$ соответственно.

Заметим, что в строках 4-й, 5-й и т. д. этой таблицы записаны ряды, расходящиеся в смысле Чезаро. Действительно, пусть в $k$-й строке $(k \geqslant 4)$ ряд сходится в смысле Чезаро, но тогда ряд в $(k-1)$-й строке должен сходиться в обычном смысле. У нас $k \geqslant 4$, т.е. $k-1 \geqslant 3$, но ряд 
в строке с номером 3 не может сходиться в обычном смысле. Для номера 3 это доказано выше, а для больших номеров это следует из тех соображений, что если ряд с таким номером сходится в обычном смысле, то сходится и ряд с номером на 1 меньшим, так как он получается переходом $C(1)$ из сходящегося в обычном смысле ряда, и так далее, так мы дойдем до третьей строки. Но ряд в этой строке сходится в обычном смысле, т.е. приходим к противоречию.

Итак, о рядах в приведенной выше таблицы мы можем утверждать следующее:

1) во второй строке таблицы записан сходящийся в обычном смысле числовой ряд;

2) в третьей строке записан ряд, не сходящийся в обычном смысле, но сходящийся в смысле Чезаро;

3) в последующих строках записаны ряды, расходящиеся и в обычном смысле, и в смысле Чезаро.

Напомним, что каждый предыдущий ряд таблицы получается из следующего за ним при помощи перехода $C(1)$.

Теперь становится понятным и решение нашей задачи. По заданному числу $N$ в качестве нужного нам ряда мы берем ряд, занимающий в таблице строку под номером $N+3$. При переходе $C(1)$ из него получается ряд, записанный в строке под номером $N+2$, при переходе $C(1)-$ ряд строки под номером $N+1$ и т. д. При переходе $C(N-1)$ получим ряд

$$
a_{41}+a_{42}+\ldots+a_{4 n}+\ldots
$$

4-й строки, который еще расходится в смысле Чезаро, а при следующем переходе $C(N)$ получится ряд

$$
a_{31}+a_{32}+\ldots+a_{3 n}+\ldots
$$

из третьей строки, который сходится в смысле Чезаро. Искомый числовой ряд, таким образом, построен.

Заметим в заключение, что существуют ряды, расходящиеся в смысле Чезаро, для которых последовательность переходов $C(1), C(2), \ldots, C(N), \ldots$ не приводит к ряду, сходящемуся в смысле Чезаро. Таким, например, будет ряд $1+1+1+\ldots$.

\section{СПИСОК ЛИТЕРАТУРЫ}

1. Харди Г. Расходящиеся ряды. - М.: ИЛ, 1951.

Тимошенко Валерий Викторович

Московский педагогический государственный университет

E-mail: vvtimoschenko@gmail.com 